\title{
60-W average power in 810-fs pulses from a thin-disk Yb:YAG laser
}

\author{
E. Innerhofer, T. Südmeyer, F. Brunner, R. Häring, A. Aschwanden, and R. Paschotta \\ Physics Department/Institute of Quantum Electronics, Swiss Federal Institute of Technology (ETH), ETH Zürich Hönggerberg-HPT, \\ CH-8093 Zürich, Switzerland
}

C. Hönninger and M. Kumkar

TRUMPF-Laser GmbH + Co. KG, Aichhalder Strasse 39, 78713 Schramberg, Germany

U. Keller

Physics Department/Institute of Quantum Electronics, Swiss Federal Institute of Technology (ETH), ETH Zürich Hönggerberg-HPT, CH-8093 Zürich, Switzerland

Received September 6, 2002

We demonstrate a passively mode-locked diode-pumped thin-disk Yb:YAG laser generating 810-fs pulses at $1030 \mathrm{~nm}$ with as much as $60 \mathrm{~W}$ of average output power (without using an amplifier). At a pulse repetition rate of $34.3 \mathrm{MHz}$, the pulse energy is $1.75 \mu \mathrm{J}$ and the peak power is as high as $1.9 \mathrm{MW}$. The beam quality is close to the diffraction limit, with $M^{2}<1.1$. (C) 2003 Optical Society of America

OCIS codes: $140.3480,140.3580,140.4050,140.5680$.

In recent years, femtosecond and picosecond lasers have been developed with ever-increasing average output power. The high peak power of such lasers is particularly interesting for applications involving nonlinear effects, e.g., wavelength conversion for laser projection displays. For reasons of simplicity and compactness it is advantageous to obtain such performance directly from a diode-pumped laser oscillator without the use of additional amplifier stages. Such lasers can be achieved by combination of a diode-pumped thin-disk laser head ${ }^{1}$ with a semiconductor saturable-absorber mirror ${ }^{2,3}$ (SESAM) for passive mode locking. As the first reported laser based on this concept, an $\mathrm{Yb}$ :YAG laser generating $16 \mathrm{~W}$ of average output power in $730-\mathrm{fs}$ pulses was demonstrated. ${ }^{4}$ More recently, $22 \mathrm{~W}$ of average power in pulses as short as $240 \mathrm{fs}$ was obtained from a passively mode-locked thin-disk laser based on $\mathrm{Yb}: \mathrm{KY}\left(\mathrm{WO}_{4}\right)_{2}$ (also called $\mathrm{Yb}: \mathrm{KYW}$ ) that was used as the laser material. ${ }^{5}$ Also, the successful application of these high-power mode-locked lasers as pump sources for optical parametric generators ${ }^{6,7}$ and a fiber-feedback optical parametric oscillator ${ }^{6,8}$ has been demonstrated.

There are other sources of mode-locked pulses with high average power. Mode-locked rod lasers have generated as much as $27 \mathrm{~W}$ of average power in 19 -ps pulses, ${ }^{9}$ and more recently, a femtosecond fiber chirped-pulse amplification system was reported that yielded $125 \mathrm{~W}$ of average power before compression. ${ }^{10}$

A crucial feature of the thin disk concept is power scalability, which arises from the special geometry of gain medium and saturable absorber. The power scalability of thin-disk lasers for high-power cw operation with good transverse beam quality was demonstrated previously. ${ }^{1}$ In this Letter we report on what is to our knowledge the first realization of power scaling of mode-locked thin-disk lasers to an unprecedented level of 60 -W average output power in 810 -fs pulses in the femtosecond regime.

In thin-disk lasers, gain is provided by a disk of laser material with a thickness that is much smaller than the spot diameter of pump and laser mode. One end face of the disk is coated for high reflectivity and attached directly to a heat sink. Thus the resulting heat flow is longitudinal and nearly one dimensional. The same applies to the SESAM, for which the laser mode diameter is also much larger than the thickness of the substrate. The output power of a given laser design can be scaled up by multiplication of the pump power and the mode areas on both gain medium and SESAM by the same factor. As a consequence of the one-dimensional heat flow in both elements, the temperature will not rise if the cooling system is capable of removing the additional heat. The effect of thermal lensing will therefore not increase. Also, the intensities remain unchanged in the laser material and on the absorber, so neither thermal nor nonthermal damage of the SESAM limits power scaling. As another consequence of the unchanged intensities and therefore the unchanged saturation of gain and absorption, the tendency toward $Q$-switched mode locking does not increase. Still, one has to face several challenges in scaling up the average power of mode-locked thin-disk lasers to such high power levels. First, the transverse mode quality has to be close to the diffraction limit as higher transverse modes would destabilize the mode locking. Thermal effects in intracavity components, e.g., for introducing negative cavity dispersion, have to be minimized for this purpose. Further, one has to avoid various mode-locking instabilities such as $Q$-switched mode locking ${ }^{11}$ and multiple pulsing caused by spatial hole burning. ${ }^{12}$ The laser cavity has to be designed such that it has appropriate mode sizes in gain medium and absorber, a suitable cavity length (which determines the pulse repetition rate), 
and minimum sensitivity to changes of the thermal lens in the gain medium and to a slight nonflatness of the SESAM, which becomes important for large mode areas.

In this Letter we report on a thin-disk Yb:YAG laser generating $60 \mathrm{~W}$ of average power in 810 -fs pulses. It is based on an improved laser head constructed by TRUMPF-Laser (Schramberg, Germany), containing a $100-\mu \mathrm{m}$ thin $\mathrm{Yb}: \mathrm{YAG}$ disk that is $\approx 10 \%$ doped. One face of the disk is coated for high reflectivity at both pump and laser wavelength, and the other side has an antireflection coating for both wavelengths. The disk is slightly wedged to eliminate effects from residual reflections from the antireflection coating. The reflecting face is directly attached to a cooling finger. We pumped the $\mathrm{Yb}: \mathrm{YAG}$ disk with as much as $370 \mathrm{~W}$ of power at $940 \mathrm{~nm}$ from diode bars. The pump optics are aligned for 16 passes (i.e., 8 double passes) through the disk to ensure efficient pump absorption despite the thinness of the disk. The diameter of the pump spot is $\approx 2.8 \mathrm{~mm}$, and the thickness of the disk is $\approx 100 \mu \mathrm{m}$. This configuration leads to only weak thermal lensing in the disk (with an estimated focusing power of $<0.3 \mathrm{~m}^{-1}$ ). The modulation depth of the SESAM is roughly estimated to be $0.5 \%$. To minimize the losses, we grew the SESAM by molecular chemical-vapor deposition. The structure of the SESAM contains a single 10-nm-thick InGaAs quantum well in a standard low-finesse structure. ${ }^{3,13}$ The relatively long recovery time (not measured) is still short enough for stable mode locking with subpicosecond pulses. ${ }^{14}$ The SESAM is mounted on a water-cooled heat sink kept at $\approx 20^{\circ} \mathrm{C}$.

For soliton mode locking ${ }^{15}$ we need sufficient self-phase modulation and negative group-delay dispersion (GDD). As the Kerr nonlinearity of the thin disk alone is too small for stable soliton pulses, we used a 1-mm-thick Brewster plate to increase the nonlinearity and to enforce linear polarization of the laser mode. The negative GDD is introduced by 11 dispersive mirrors, generating a total GDD of $\approx-22,000 \mathrm{fs}^{2}$ per round trip. These mirrors have been produced by Layertec, Germany, and have been optimized for small absorption and thus for weak thermal effects.

With the setup shown in Fig. 1, we obtain selfstarting mode locking with an average output power of $60 \mathrm{~W}$ (with a pump power of $310 \mathrm{~W}$ ) in pulses of $810-\mathrm{fs}$ duration at center wavelength of $1030 \mathrm{~nm}$ (Fig. 2). The transmission of the output coupler is $9.0 \%$. The pulse repetition rate is $34.3 \mathrm{MHz}$, leading to an output pulse energy of $1.75 \mu \mathrm{J}$ and a peak power as high as 1.9 MW. The time-bandwidth product is 0.34 , which is slightly larger than the ideal time-bandwidth product for soliton pulses $(0.315)$. The achieved beam quality is close to the diffraction limit, with an $M^{2}$ value measured to be below 1.1 .

A previous cavity setup of this laser was operated with only $-9400 \mathrm{fs}^{2}$ intracavity dispersion, a lower output coupler transmission, and a smaller beam radius on the Brewster plate. It was designed so that it would make possible the generation of $800-\mathrm{fs}$ solitons, assuming the calculated Kerr nonlinearity.
However, in this configuration operated at full power the laser generated bunches of three or four pulses with equal energy, spaced by typically 0.7 to $0.8 \mathrm{~ns}$, as was seen with a fast sampling oscilloscope. In this multiple pulsing regime, the autocorrelation, the sampling scope signal, and a photodiode signal observed with a microwave spectrum analyzer appeared to be as stable as in the single-pulse regime. With lower pump power, fewer pulses or even a single pulse with similar energy was observed. We then managed to obtain single pulses at full power by exchanging some of the dispersive mirrors for others with more negative dispersion. These findings suggest that we had soliton pulses in this laser, but there was a significant additional nonlinear phase shift that appears to be contributed by the mirrors. (Note that for a typical beam radius of the order of $0.6 \mathrm{~mm}$ the intracavity peak intensity is $\approx 3.7 \mathrm{GW} / \mathrm{cm}^{2}$.) The findings also explain why we observed only a slight change of the pulse duration when we moved the Brewster plate to a position in the cavity where the beam radius was considerably smaller: Apparently only a smaller part (roughly one-sixth) of the total nonlinear phase shift $(\approx 0.1 \mathrm{rad})$ comes from the Brewster plate. The Yb:YAG disk does not significantly contribute to the Kerr nonlinearity because of its small thickness and large mode radius.

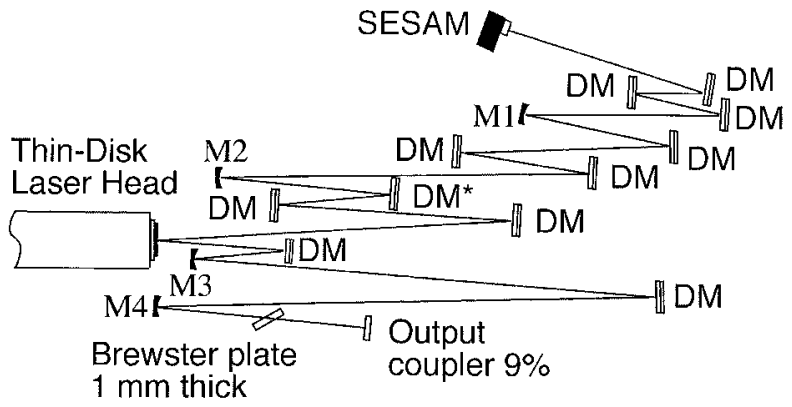

Fig. 1. Setup of the Yb:YAG thin-disk laser for the 810-fs pulses: M1-M4, spherically curved mirrors; DMs, dispersive mirrors with $-1050 \mathrm{fs}^{2}$ per bounce; $\mathrm{DM}^{*}$, dispersive mirror with $-540 \mathrm{fs}^{2}$ per bounce.

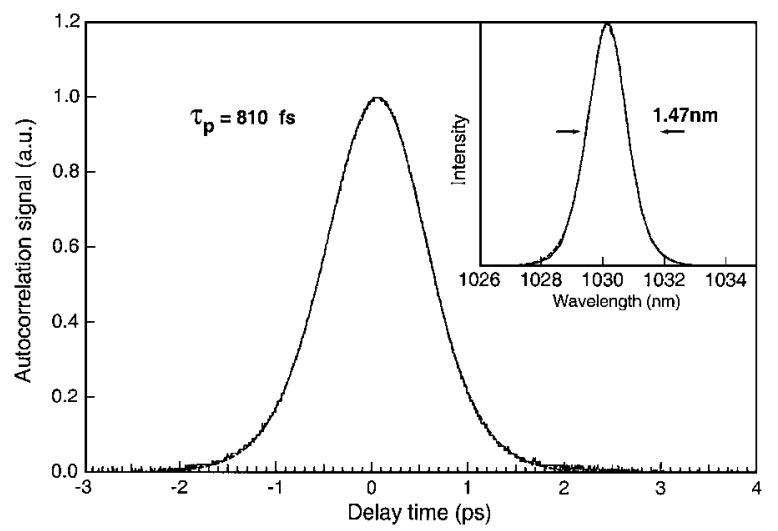

Fig. 2. Autocorrelation trace and optical spectrum (inset) of the 810-fs pulses obtained from the thin-disk Yb:YAG laser at $60-\mathrm{W}$ average output power. The dashed curves, representing $\operatorname{sech}^{2}$ fits, overlap the data well. The time-bandwidth product is 0.34 . 
To confirm single-pulse operation in the final setup (in addition to the measurement with the sampling oscilloscope), we measured the efficiency of secondharmonic generation in a critically phase-matched 5-mm-long $\mathrm{LiB}_{3} \mathrm{O}_{5}$ crystal. We did this measurement with the output beam of the laser attenuated to a few watts in the regime with low conversion efficiency. The measured efficiency is in reasonable agreement with the expectation for single-pulse operation, whereas in earlier cases with multiple pulsing the efficiencies were significantly lower because of the reduced peak powers.

The mode radius on the SESAM is $0.85 \mathrm{~mm}$, leading to an incident pulse fluence of $0.86 \mathrm{~mJ} / \mathrm{cm}^{2}$, which is an order of magnitude above the estimated saturation fluence. Excessive thermal effects from the SESAM have not been observed, but there seem to be some thermal lensing effects in the dispersive mirrors (particularly for earlier designs), making the alignment somewhat more difficult than with normal highly reflecting mirrors. These thermal effects, together with the above-mentioned additional nonlinear phase shift, are currently the limiting factors for further power increase. Apart from further improvements of dispersive mirrors for high-power operation, a significant improvement may also be achieved by use of a laser cavity with four passes through the disk ${ }^{16}$ which will allow us to reduce the intracavity average power and peak intensity by a factor of 2 .

In conclusion, we have obtained $810-\mathrm{fs}$ pulses with up to $60 \mathrm{~W}$ of average power from a passively mode-locked thin-disk Yb:YAG laser. To our knowledge, these powers are the highest ever reported directly from a mode-locked laser. We will further investigate the effects that currently limit the output power and contribute additional nonlinearity.

E. Innerhofer's e-mail address is innerhofer@ phys.ethz.ch.

\section{References}

1. A. Giesen, H. Hügel, A. Voss, K. Wittig, U. Brauch, and H. Opower, Appl. Phys. B 58, 363 (1994).

2. U. Keller, D. A. B. Miller, G. D. Boyd, T. H. Chiu, J. F. Ferguson, and M. T. Asom, Opt. Lett. 17, 505 (1992).
3. U. Keller, K. J. Weingarten, F. X. Kärtner, D. Kopf, B. Braun, I. D. Jung, R. Fluck, C. Hönninger, N. Matuschek, and J. Aus der Au, IEEE J. Sel. Top. Quantum Electron. 2, 435 (1996).

4. J. Aus der $\mathrm{Au}, \mathrm{G}$. J. Spühler, T. Südmeyer, R. Paschotta, R. Hövel, M. Moser, S. Erhard, M. Karszewski, A. Giesen, and U. Keller, Opt. Lett. 25, 859 (2000).

5. F. Brunner, T. Südmeyer, E. Innerhofer, R. Paschotta, F. Morier-Genoud, J. Gao, K. Contag, A. Giesen, V. E. Kisel, V. G. Shcherbitsky, N. V. Kuleshov, and U. Keller, Opt. Lett. 27, 1162 (2002).

6. T. Südmeyer, J. Aus der Au, R. Paschotta, U. Keller, P. G. R. Smith, G. W. Ross, and D. C. Hanna, J. Phys. D 34, 2433 (2001).

7. T. Südmeyer, F. Brunner, R. Paschotta, U. Keller, T. Usami, H. Ito, M. Nakamura, and K. Kitamura, in Conference on Lasers and Electro-Optics, Vol. 73 of OSA Trends in Optics and Photonics Series (Optical Society of America, Washington, D.C., 2002), paper CTuO4.

8. T. Südmeyer, J. Aus der Au, R. Paschotta, U. Keller, P. G. R. Smith, G. W. Ross, and D. C. Hanna, Opt. Lett. 26, 304 ( 2001).

9. G. J. Spühler, T. Südmeyer, R. Paschotta, M. Moser, K. J. Weingarten, and U. Keller, Appl. Phys. B 71, 19 (2000).

10. A. Liem, J. Limpert, T. Schreiber, S. Nolte, H. Zellmer, A. Tünnermann, V. Reichel, S. Unger, S. Jetschke, and H.-R. Müller, in Advanced Solid-State Lasers, Vol. 68 of OSA Trends in Optics and Photonics Series (Optical Society of America, Washington, D.C., 2002), p. 128.

11. C. Hönninger, R. Paschotta, F. Morier-Genoud, M. Moser, and U. Keller, J. Opt. Soc. Am. B 16, 46 (1999).

12. R. Paschotta, J. Aus der Au, G. J. Spühler, S. Erhard, A. Giesen, and U. Keller, Appl. Phys. B 72, 267 (2001).

13. C. Hönninger, G. Zhang, U. Keller, and A. Giesen, Opt. Lett. 20, 2402 (1995).

14. R. Paschotta and U. Keller, Appl. Phys. B 73, 653 (2001).

15. F. X. Kärtner, I. D. Jung, and U. Keller, IEEE J. Sel. Top. Quantum Electron. 2, 540 (1996).

16. F. Brunner, R. Paschotta, J. Aus der Au, G. J. Spühler, F. Morier-Genoud, R. Hövel, M. Moser, S. Erhard, M. Karszewski, A. Giesen, and U. Keller, Opt. Lett. 26, 379 (2001). 